\title{
The relationship between watching sport events and spectators' engagement in physical activities
}

\author{
Palik M. ${ }^{1 \mathrm{ABCDE}}$, Abdi K. ${ }^{2 \mathrm{ABCDE}}$ \\ ${ }^{1}$ Faculty of Physical Education and Sports Sciences, University of Tehran, Iran \\ ${ }^{2}$ Ferdowsi University of Mashhad, Iran
}

Authors' Contribution: A - Study design; B - Data collection; C - Statistical analysis; D - Manuscript Preparation; E - Funds Collection

\begin{abstract}
Purpose: $\quad$ The purpose of the present research was to investigate the relationship between watching sport events in stadiums and spectators' physical activities.

Material: $\quad$ The population included spectators of basketball, volleyball, and handball in Tehran stadiums, which is estimated to be more than a hundred thousand people. The sample consisted of 384 spectators who were chosen using the Morgan Table in the simple random method, and they filled the questionnaire before the games started. The instrument was a researcher-developed questionnaire whose validity was confirmed by sports management and communication experts. The reliability was obtained to be 0.86 using Cronbach's alpha test. The collected data was analyzed using K-S test, chi-square test, Spearman correlation coefficient (using SPSS software), and confirmatory factor analysis (using LISREL 8.8 software).

Results: $\quad$ The results indicated that there is a significant positive relationship between watching sport events in stadiums and spectators' intention to do physical activities. Also, there was a significant positive relation between effective factors on spectator during watching sport events and doing physical activities. These factors are related to the stadiums, sport events and social interrelation.

Conclusions: It seems that watching sport events and the encouraging factors in sporting environment and stadiums promotes physical activity and health. Since the present research focuses on an important social effect of sport events, it can be a start point for researchers to discover if encouraging people to watch sport events is a way to maintain social health.

Keywords: physical activity, spectator, stadiums, sports.
\end{abstract}

\section{Introduction}

All the advances such as electronic and credit cash, online trade, humanoid robots, and hundreds of other achievements have made our lives easier, and modern technology made humans inactive like the machines. The result is the emergence of a variety of diseases, including heart-respiratory diseases. Sports Medicine scientists believed that not getting fifteen minutes of physical activity with no sweat at least once in a day will result in motor poverty [1]. Therefore, changing from a passive to an active lifestyle can reduce the risk of many diseases [2]. Meanwhile, the solution to these problems is taking up physical activities, especially sports. A healthy body is the most basic of human needs. Without healthy body, it is not possible to nurture the human personality and spirituality, and the mental health will be in jeopardy. By investing in sports activities, while reducing health care costs and increasing productivity in society, the mental and physical health will improve, too [3].

Public sports are considered as one of the indexes of human development in the communities because they increase life expectancy through the promotion of public health [4]. Public sport and quantitative recreation objectives are based on increasing motivation and social awareness about the role of sport in life. It also concerns the allocation of a portion of the household expenditure basket to sports [5]. In their model, Mull (2005) claim that the development of educational sports and public

(C) Palik M., Abdi K., 2018

doi:10.15561/18189172.2018.0607 sports will increase public participation. If professional sports are developed, smaller groups can participate in them, and they will often watch them. What is clear is that a countless number of people interested in sports and physical activities make up spectators [6]. Spectators are people who watch physical activities and sports events for fun, entertainment, excitement, and joy. Due to their differences in behavioral characteristics, they will show different responses and reactions to different sports events in different conditions and environments [7].

Nowadays, watching sports events among individuals has increased more than ever before compared with participation in sports activities. For example, few people participate in sports as active participants, but inactive participants (spectators) can be seen in many fields. These people watch sports event as spectators note being aware of the fact that they can also take part in their favorite sports activities [8]. Spectators who spend on sports, purchase tickets, travel to watch tournaments live in stadiums will consequently be of more value and importance than those who watch the tournaments at home. Culture has a significant impact on the attendance of people in stadiums to watch sports events and participation in sports activities. Education is an important cultural factor in this regard [9]. In addition to cultural factors, socioeconomic factors also affect people's attendance in tournaments and their participation in sports activities [10].

Spectators choose their favorite sports based on their interests, experience, and familiarity with the sport. They participate in matches in which both teams 
are already known to them because they feel they can easily identify with them [11]. Also, Torkfar, in his research entitled "motivational factors influencing spectators' attractiveness to the Iranian soccer league", showed that the correlation between internal factors and attractiveness of spectators was 0.99 . According to the presented functional pattern, the effective internal factors in attracting spectators included interest, beauty, stress, expression of personal feelings, escaping from the problems of life, fun, role playing by players, knowledge and information, motivation, attitudes and beliefs. Beauty had the greatest and attitudes and beliefs had the smallest impact on directly attracting spectators. The correlation of external factors with attracting spectators was 0.98 . The effective external factors in attracting spectators included culture, social activism, community pride, family, friends, and marketing. Marketing had the greatest and social activism had the smallest impact on directly attracting spectators. Spectators create a close relationship and mutual affection with their teams and athletes at stadiums. Does this relationship result in motivating them to participate in their favorite sports activities? Motivation is a dynamic action and a relationship between human needs and the actions that creates motion and activity in people through developing tension and pressure. It then makes people behave in a purposeful way that results in the satisfaction of their needs [12].

Internal factors are related to the motivation of the spectators to watch a sport event closely and the degree of spectator's willingness to exercise. External factors on spectators during watching tournaments include such influencing factors as environmental and human factors [13]. People who have are highly motivated to exercise and commit themselves to physical activities learn to have an active lifestyle and make themselves healthier. In terms of physical power, they are also better prepared to perform daily tasks [14]. Weiss has shown that factors that influence participation in sport activities include health and wellness, enhancing the ability of the body, learning new skills, making a friendly relationship, admission to peer groups, support from others, and the joy arising from positive participation and minimal negative experience associated with physical activities [15].

It can be seen that several factors that may influence participation in physical activity have been studied. It is interesting that research has neglected sport itself in general and watching it in particular. Regarding the impact of factors such as health, social relationships, and pleasure the question arises as to whether spectators engage in exercising in addition to watching sports events or just make up the group who are only interested in watching sports events? What internal and external factors will attract spectators to physical activities? The present study aims to examine the relationship between watching sport events in stadiums and spectators' engagement in physical activity to offer desirable solutions for the further engagement of spectator's in different sports activities through obtaining comprehensive information and considering the needs.

\section{Materials and Methods}

Participants.

The population included all spectators of basketball, volleyball, and handball in Tehran stadiums whose number was estimated to be more than a hundred thousand people based on the nominal capacity of the venues and stadiums. The sample consisted of 384 spectators who had been chosen using the Morgan Table in a simple random method.

\section{Research Design}

The present study was an applied study in terms of purpose, descriptive of the correlational type in terms of strategy, and a survey in terms of conduction which was conducted as a field study. The instrument was a researcher-developed questionnaire that was set based on the objectives of the present study after studying Zhang et al (2001) questionnaires in investigating factors influencing the attendance of spectators in stadiums for ball sports and Torkfar (2009) standardized questionnaire on spectators' motivation to attend stadiums $[11,16]$. This questionnaire included 27 questions on a 5-point Likert scale ranging from strongly agree to strongly disagree, as well as questions on demographic factors. After the initial stages, it was presented to 13 experts and specialists in the field of sports management to assess its validity. After applying the comments of the experts to ensure the validity of the questionnaire, a pilot study was done on 30 spectators of Mahram and Saman Bank basketball teams at Azadi stadium for more information about the reliability of the instrument. Test results showed a Cronbach's alpha of 0.86 which indicates a high reliability for the instrument. The questionnaire distributor team attended the desired field of tournament venues. The questionnaires were distributed among the spectators and were then collected. Members of the distribution team had been trained by the researchers, and the required permissions had been obtained from the organizers after taking legal measures.

Statistical Analysis

Mean, standard deviation, and frequency were used in the descriptive statistics section. In inferential statistics section, Kolmogorov-Smirnov test, chi-square test, Spearman correlation coefficients (using SPSS), and confirmatory factor analysis (using the LISREL software) were used. All the analyzes were performed in the 95 percent confidence level.

\section{Results}

According to the above table, the average age of participants in the study was 27 years, and the number of men and women were equal. Most of the participants were single (78.2 percent). Most of the participants sometimes attended stadiums to watch sports events $(53 \%)$. The majority of the participants $(38 \%)$ were physically active for three days a week. The maximum Time Spent to follow up sports events was for people who had been watching sports events in sport facilities for more than 5 years $(42.2 \%)$.

Based on Table 2, the results show that the data is not normally distributed at the 0.05 level. Therefore, 
nonparametric tests should be used for the inferential analysis of the data.

The results presented in Table 3 show that the observed frequencies for the 11 questions on the motivation for attendance differ from the expected frequencies, and the difference is statistically significant. Therefore, the motivations of spectators to attend stadiums was significantly high among the participants.

Two methods were used to test the main hypotheses of the study. Given the ordinal nature of the questionnaire scales, Spearman correlation test was used. Also, given the multiplicity of variables in the questionnaire and the fact that several questions existed for the same variable, a confirmatory factor analysis was used as a complementary test. The results of the analysis of the research hypotheses are presented below.

The information from Table 4 shows that there was a significant relationship between watching sports events in stadiums and spectators' physical activities, given the obtained significant level. The observed correlation coefficient $(\mathrm{R}=0.20)$ indicates a positive direct correlation between attending stadiums and spectators' physical activity.

The information from Table 5 shows that there was a relationship between the effective factors on spectators during watching and the spectators' physical activity, given the obtained significance level $(0.0001<0.05)$. The observed correlation coefficient $(\mathrm{R}=0.342)$ indicates a

Table 1. Descriptive statistics for the demographic factors

\begin{tabular}{llll}
\hline Age & Mean & SD & Range \\
& $\mathbf{2 7}$ & $\mathbf{7 . 7 7}$ & $\mathbf{5 4}$ \\
\hline Gender & Frequency & $\begin{array}{l}\text { frequency } \\
\text { percent }\end{array}$ & $\begin{array}{l}\text { Cumulative frequency } \\
\text { percent }\end{array}$ \\
Woman & 192 & 50 & 50 \\
Man & 192 & 50 & 100 \\
Total & 384 & 100 & \\
Marital Status & & & \\
Single & 297 & 77.3 & 77.3 \\
Married & 83 & 21.6 & 100 \\
Total & 380 & 100 & \\
Attending Status in sports venues for watching & & & \\
tournaments & & & \\
Rarely & 64 & 16.8 & 16.8 \\
Sometimes & 202 & 53.0 & 69.8 \\
Always & 115 & 30.2 & 100 \\
Total & 381 & 100 & \\
Weekly physical activity & & & 9.2 \\
I do not work & 35 & 9.2 & 33.5 \\
One day a week & 93 & 24.3 & 71.5 \\
Three days a week & 145 & 38.0 & 100 \\
Every day & 108 & 28.3 & \\
Total & 382 & 100 & 17.5 \\
Time Spent to watch sports events & & & 39.5 \\
Less than a year & 66 & 17.5 & 100 \\
One to three years & 83 & 22.0 & \\
Three to five years. & 69 & 18.3 & \\
More than five years & 159 & 42.2 & \\
Total & 377 & 100 & \\
\hline
\end{tabular}

Table 2. Test of normality for the distribution of the data

\begin{tabular}{llll}
\hline Test & KS & & \\
Variables & Statistics & Degrees of freedom & Significance level \\
\hline Motivations & 0.060 & 384 & 0.0001 \\
Watching & 0.103 & 384 & 0.001 \\
Participation & 0.162 & 384 & 0.0001 \\
Effective factors & 0.147 & 384 & 0.0001 \\
\hline
\end{tabular}


positive direct correlation between the effective factors on spectators during watching and the spectators' physical activity.

In the following figure, the results of the estimated correlation between the main variables (shown as ovals) using the confirmatory factor analysis are shown. Rectangles inside the figure represents questions of the questionnaire.

Figure 1 marked the amount of the covariance between the main study variables in a standardized form. Fitness Indicators at the bottom of Figure 1 show the fitness of the model for extracting relationships. It should be noted that the specified fitness parameters have been approved with the assumption that the components related to each of the variable presented in the figure on the left are related to each other, which seems reasonable.

In Figure 2, significance of the coefficients is given by applying the $t$ values. Coefficients larger than +1.96 or less than -1.96 are considered significant. As can be seen, all the coefficients in Figure 1 are statistically significant.

Regarding the motivations, the most important motivation is related to question s7 (supporting one's favorite team) with a factor loading of 0.56 , as shown in the figure. The second major motivation is related to question s9 (feeling proud) with a factor loading of 0.55 , and the third most important motivation is related to question s5 (more communication with the players) with a factor loading of 0.53. All the factor loading values in Figure 2 that are related to the $t$ significant values are shown to be significant. The lowest motivation of the spectators to attend stadiums is related to s3 (Entertainment) with a factor loading of 0.30 . As it has been specified, the coefficient of variance (which is equivalent to the correlation) between watching tournaments in stadiums and spectator's participation in physical activity is equal to 0.19 . $t$ value is equal to 3.01 , which is greater than +1.96 , hence significant. Thus, the hypothesis of the existence of a relationship between watching tournaments in stadiums and participation in physical activities is confirmed. Also, the coefficient of variance between the effective factors on the spectators during watching tournaments in stadiums and spectators' participation in physical activities is 0.42 , and the $t$ value is equal to 7.67 , which is larger than +1.96 , hence significant. Therefore, the hypothesis that there is a relationship between effective factors on spectators during watching the tournaments and participating in physical activities is confirmed. Both of these sets of results were confirmed by applying Spearman's test, and the relationship between "watching tournaments in stadiums" and "effective factors on the spectators during watching" with "spectators' participation in physical activity" was significant.

\section{Discussion}

The correlation test showed that there is a significant direct positive relationship between watching sports events at stadiums and spectators' physical activities. It means that the more spectators watch sports events the more they participate in physical activities. Also, applying a confirmatory factor analysis indicated that covariance

Table 3. The results of chi-square test for the motivations to watch tournaments in sporting venues

\begin{tabular}{llll}
\hline The presence Motivations & Chi-Square & Degrees of freedom & Significant level \\
\hline Attractiveness & 351.5 & 4 & 0.001 \\
Stress and mental pressure reduction & 155.7 & 4 & 0.001 \\
Entertainment & 105.8 & 4 & 0.001 \\
Vitality increasing & 305.9 & 4 & 0.001 \\
More communication with players & 296.6 & 4 & 0.001 \\
belonging in peer groups & 93.2 & 4 & 0.001 \\
Support your favorite team & 170.5 & 4 & 0.001 \\
Meeting with new people & 120.3 & 4 & 0.001 \\
Feel Proud & 219.2 & 4 & 0.001 \\
Staying away from monotony & 102 & 4 & 0.001 \\
Activities Background & 125.9 & 4 & 0.001 \\
\hline
\end{tabular}

Table 4. The relationship between watching sports events in the stadium and spectators' physical activity using Spearman correlation test

\begin{tabular}{lll}
\hline number & Spearman Correlation coefficient & Significant level \\
\hline 384 & 0.20 & 0.0001 \\
\hline
\end{tabular}

Table 5. The correlation between effective factors on spectators during watching and spectators' physical activity using Spearman correlation test

\begin{tabular}{lll}
\hline number & Spearman Correlation coefficient & Significant level \\
\hline 384 & 0.342 & 0.0001 \\
\hline
\end{tabular}




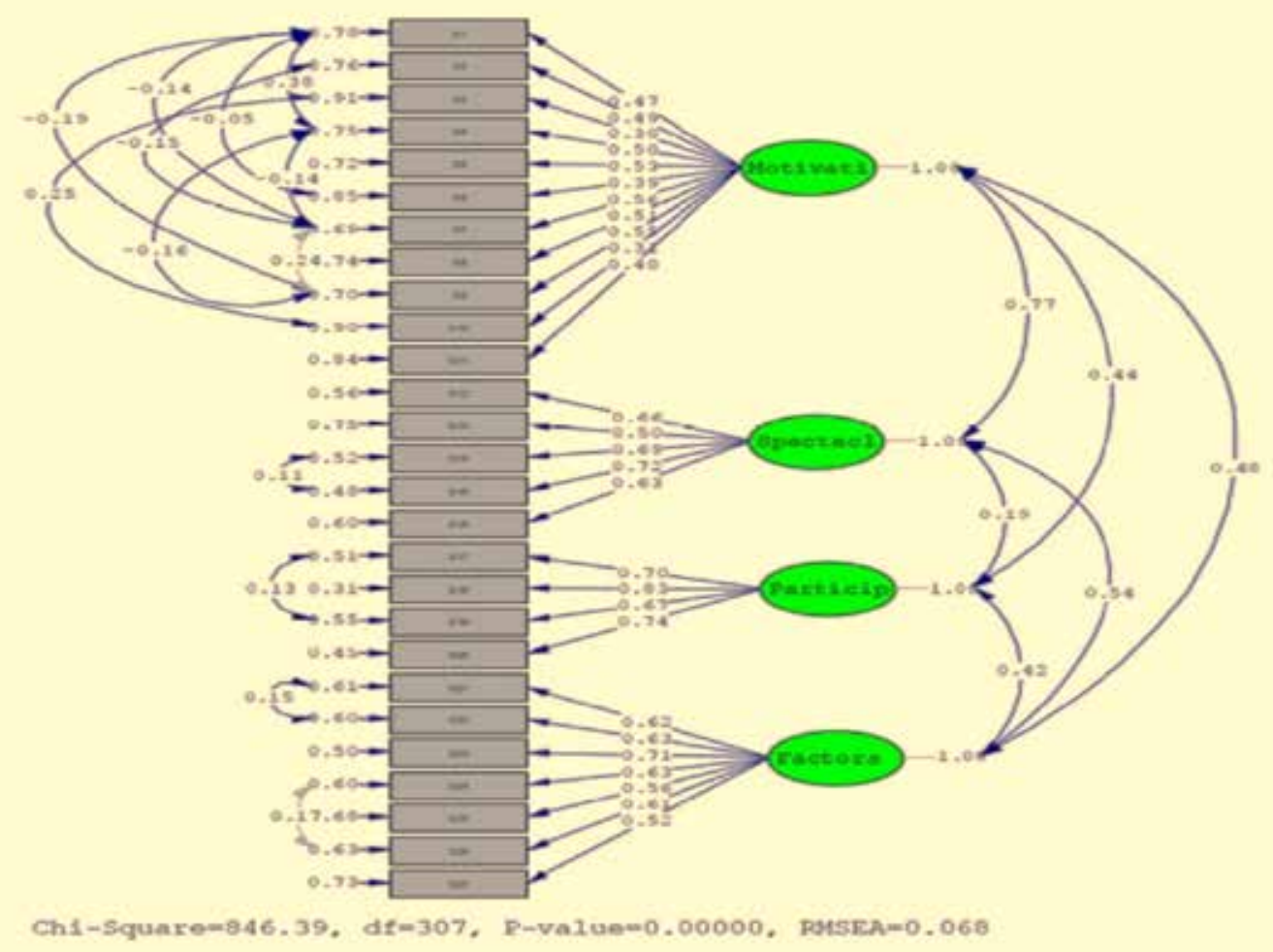

Figure 1. A confirmatory factor analysis of the main study variables with covariance coefficients between them (standardized coefficients)

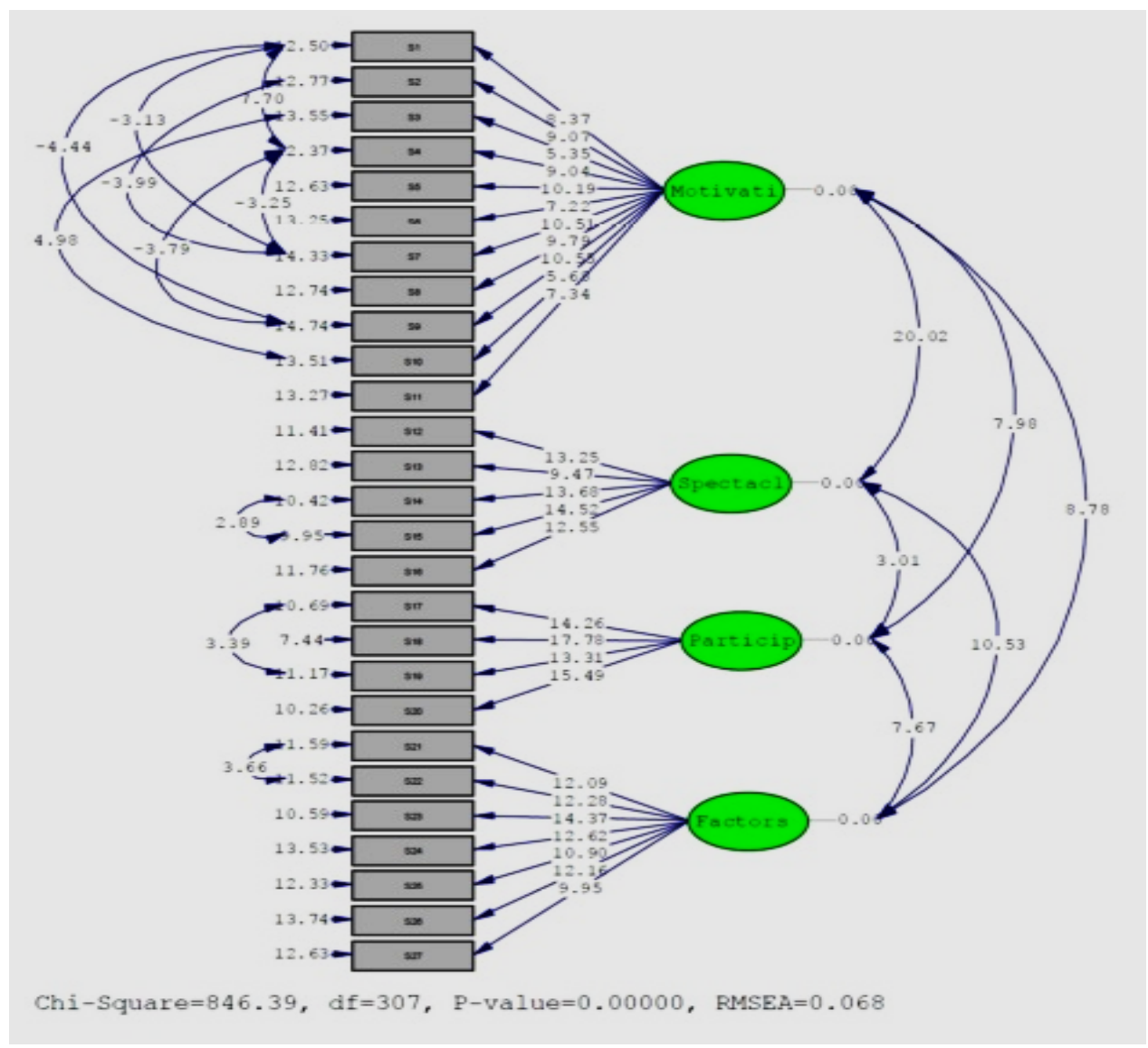

Figure 2. The $t$ values of confirmatory factor analysis for the main study variables 
coefficient (which is equal to correlation) between watching sports events at stadiums and participation in physical activities is equal to 0.19 . Also the t-value is equal to 3.01, which is larger than +1.96 , hence significant. It can be said that physical activity and exercising have an efficient interaction with such environmental factors as social, cultural, and personal forces, which altogether shape an individual's characteristics. Another category of physical activities has a relationship with forces that represents functional form of the physical organizations, among which physical activity and sports are of greater importance because they emerge from the relationship between the individual and culture and society.

In the third category, physical activity is related to forces that are effective in spreading or limiting physical activities. This forces are related to the surrounding natural environment as well as to social institutions such as economy, government, and politics as efficient inhibiting forces of society [17]. These factors have been considered in this study. As the results showed, the majority of those people who attend stadiums were people with high levels of education and income. It seems that cultural and social forces resulting from attending stadiums and watching tournaments and also the individuals themselves are inclined towards sports and physical activities. It is believed that watching sports can play a role in publicizing sport as an effective and active social institution in today's growing and changing modern society. Continuity and persistence on sports will be effective in the promotion of material and spiritual culture of the society and ensure the community's physical and mental health. Although there have not been any studies that directly address the relationship between watching sports events and physical activities, the results of the above hypothesis are consistent with the results of Ramezani Nejadet et al. (2009), Safania (2002), Funk and James (2004), and Lee et al. (2009) studies on factors influencing physical activity and sports. Some of these factors were mentioned in present study as motivation for attending stadiums [18-21]. Mehus (2005) and Wilson (2002) believe that participation in watching and playing sports is a social activity, and that people's amount of attendance originates from their economic and cultural background $[9,10]$. Also, Funk and James (2004) stated that the individuals' attitude toward sports and their dependency toward and interest in sport teams affect the amount of time they spend on attending physical activity and sports. This attitude is initially formed under the influence of family, friends, media and advertising, and success of the sports teams. Then, attractive features of a sporting environment (convenient location, quality of services, amenities and facilities of the stadiums, etc.) play a role in shaping the attitude [20].

The correlation test showed that there is a significant direct positive relationship between the effective factors on spectators during watching sports events and spectators' physical activity. It means that the greater the effective factors on spectators during watching sports events, the more they participate in physical activities. Also, applying a confirmatory factor analysis indicated that covariance coefficient (which is equal to correlation) between effective factors on spectators during watching sports events and their participation in physical activity is equal to 0.42 , and the t-value is equal to 7.67 , which is larger than +1.96 , hence significant. Thus, there is a significant relationship between effective factors on spectators during watching sports events and their participation in physical activities. Effective factors on spectators during watching tournaments such as environmental factors (rules and regulations have been implemented during the tournament, discipline and organization of tournament , suitable parking facilities, comfortable seating, quality of the billboard, etc.), organizational factors (functional and formal decisions during the game, the judgment, larger numbers of spectators, etc.), and human factors (coach's behavior, phenotype and physical abilities of the players, the technique of the team's stars, team effort, and team behavior) all can be related to spectators' attitudes toward sports activities. It seems that if effective factors such as environmental, organizational, and human factors are within an acceptable range - if facilities are considered desirable, if tournament managers have taken organizational factors to a desirable extent by applying appropriate strategies, if players and coaches behave in an acceptable and suitable way - spectators' behavior will improve and thus a culture of correct behavior will develop. Consequently, spectators who idolize the athletes will engage in sports under the influence of the above-mentioned factors, and this will result in the institutionalization of sports in society. The results of the present hypothesis is consistent with Kialashaki (2008), Nicholson and Hui (2005), Hooi and Yusof, (2008), and Hall; Mahony and Vieceli (2010) studies [13, 22-24]. Kialashaki (2008) stated that issues such as judgment, failure in competitions, the behavior of the rival sports teams, competition sensitivity, stimulating and exciting tournaments are among the effective factors on the behavior of their spectators during the tournament [22]. Nicholson and Hoye (2005) stated that organizational and environmental effective factors on spectators during watching sports events include environmental factors (settings, stages and levels of competition, strategies of sports organizations, interactions between authorities, coaches, and players during sports tournaments) and organizational factors (functional and formal decisions during the game, refereeing, the number of spectators over the capacity) [13]. Hooi and Yusof (2008) and Lee \& Macdonald \& Wright (2009) showed that there is a significant relationship between stadium environment and the quality of team with the spectators' satisfaction and their willingness to participate in a tournament [21, 23]. Hall et al (2010) also suggested that the creation of entertaining and exciting conditions and suitable facilities such as easy access to stadium seats and parking areas are effective on the sports events spectators [24]. The results from the statistical analyses showed that all motivations of spectators' for attending stadiums are significant. These motivations include attractiveness, stress and mental pressure reduction, entertainment, increasing 
vitality, further communication with players, belonging in peer groups, supporting one's favorite team, meeting new people, feeling proud, being away from monotony, and activity background. As shown in Figure 2, the most important motivation is related to the question of S7 (supporting your favorite team) with a factor loading of 0.56 . The second most important motivation is related to the question S9 (feeling proud) with a factor loading of .55 , and the third major motivation is related to question S5 (further communication with players) with a factor loading of 0.53 . The next most important motivations were meeting new people with a factor loading of 0.51 , increasing vitality with 0.50 , stress and mental pressure reduction with $0.49,0.47$ for attractiveness, activities Background with 0.40 , Belonging in peer groups with 0.39 , being away from monotony with 0.31 . The lowest motivation for the spectators' attendance in stadiums is related to question S3 (entertainment) with a factor loading of 0.30 . According to the results, most sports spectators are motivated by "supporting one's favorite team", "feeling proud" and "further communication with players" motivations to attend in stadiums. The motivation factor "entertainment and spending leisure time in the stadiums" have the least importance.

\section{Conclusions}

The results of the statistical tests showed that there is a significant relationship between watching sports events in stadiums and doing sports activities by spectators.

\section{References}

1. Jalali Farahani M. Management of leisure and recreational sports. Tehran University Press; 2008.

2. Sharifi G. Effects of a specific exercise program on cardiovascular risk factors and comparison with one of conventional exercise programs in the morning. Olympic Quarter, 2000; 3:3-4.

3. Sanei S. Fitness and its relationship with depression and mental health among faculty members of the military universities. Islamic Azad University, Science and Research Branch; 2004.

4. Roshandel T. Explaining the role of the media in establishing the public sports in the country. Motion Magazine, 2007; 10:33-40.

5. Organization of Physical Education. Strategic document of the comprehensive system of physical education and sports development. Council of Ministers meeting; 2003.

6. Mull R. Recreational sport management. Human Kinetics Pub.; 2005.

7. Mahoney D, Nakazawa M, Funk D, James J, Gladden J. Motivational influencing factors on the behavior of league spectators. Sport management review, 2002; 5(1): 1-24. https://doi.org/10.1016/S1441-3523(02)70059-4

8. Ghasemi H. Media activities in sport. Bamdadeh Ketab Publication; 2008.

9. Mehus Ingar. Distinction through sport consumption: spectator of soccer, basketball, and ski-jumping. Norwegian university of science and technology, Norway. International review for the sociology of sport, 2005; 40(5): 132-141.

10.Wilson C. The paradox of social class and sports involvement Florida Atlantic University. Sociology of sport, 2002;1:5-16.

11.Torkfar A. Effective motivational factors on spectators'
It seems that watching sports events and the positive factors in sports environments and stadiums result in increased levels of physical activity, vitality, and health in spectators as people who are interested in sports activities. On the community level, in addition to the development of culture, an increase in correct behaviors results in the institutionalization of sports culture, vitality, and, as a result, community health. Given the evidence on the spectator-attracting nature of professional sports and given the existing ambiguities on the relationship between watching sports events and such health complication as obesity and inactivity [6]. the results of the present study deserve being paid attention to. Given the psychological and social differences in inclination towards individual and social sports, future studies can focus on individual sports. Researchers can also investigate the type of sports that spectators trend tend to follow or avoid after watching a special sports tournament. For instance, does watching high-collision sports such as boxing and wrestling develop interest or disgust in spectators? Also, a comparison can be made between the new delicate and fancy sports (like curling, Frisbee, ice skating, etc.) and high-collision professional sports (even professional football in which players sometimes injure their opponents on purpose).

\section{Conflict of interest}

The authors declare that there is no conflict of interest.

attraction to Iranian football league clubs. Faculty of Physical Education, Islamic Azad University, Tehran Central Branch; 2009.

12.Naeli M. Motivation in organizations. First Edition, Martyr Chamran University; 1994.

13.Nicholson M, Hoye R. Contextual factors associated with poor sport spectator behaviour. Managing leisure, 2005; 10: 94-105. https://doi.org/10.1080/13606710500146175

14.Koski P. Physical activity relationship (PAR). International review for the sociology of sport, 2008; 43: 151-158. https://doi.org/10.1177/1012690208095374

15.Weiss MR, Chaill BR, Pear AJ. Intensive participation in children's sport. Psychology effects of intensive sport participation on children and youth. Self-esteem, 1993; 1:3969.

16.Zhang JJ, Pease DG, Lam ET. Variables influencing the spectator decision to attend NBA game. Sport Marketing Quarterly, 2001; 5(1): 29-39.

17.Anvar Alkhuly A, Sheikhi H. Sports and society. Islamic Research Foundation of Razavi Astan Quds and SAMT, Mashhad and Tehran, 2001.

18.Ramezani Nejad R, Rahmany Nia F, Taqavi Takyar A. A study of Motivations of participants in the open spaces public sports. Journal of Sport Management, 2009; (2):19-5.

19.Safa Nia A. Assessment of student participation in sports activities. Research Project, Islamic Azad University, Sari Branch; 2001.

20.Funk D, James J. The fan attitude network (FAN) model: Exploring attitude formation and change among sport consumers. Griffith University. Sport management review, 2004; (7): 1-26. https://doi.org/10.1016/S1441-3523(04)70043-1 
21.Lee J, Macdonald D, Wright J. Young men's physical activity choices: The impact of capital, Masculinities and location. Journal of sport and social issues 2009; (33): 50-59. https://doi.org/10.1177/0193723508328904

22.Kialashaki R. Assessment of aggression in sports arenas from the viewpoint of the spectators, athletes, and physical education experts. [PhD thesis] University of Guilan; 2008.
23.Hooi SL, Yusof A. Spectator Perceptions of Physical Facility and Team Quality: A study of a Malaysian super league soccer tournament. Journal of international studies, 2008; 8: 100-107.

24.Hall J, Mahony B, Vieceli J. An empirical model of attendance factors at major sports events. Australia International Journal of hospitality management, 2010; 29: 328-334. https://doi.org/10.1016/j.ijhm.2009.10.011

\section{Information about the authors:}

Palik M. (Corresponding author); A Master of Sport Injury and Corrective Exercises; http://orcid.org/0000-0002-3019-5350; teamworkir1@gmail.com; Faculty of Physical Education and Sports Sciences, University of Tehran; Tehran, Enghelab Square, Iran.

Abdi K.; Ph.D. Candidate in Sports Management; http://orcid.org/0000-0002-7505-9934; goodkambiz@gmail.com; Ferdowsi University of Mashhad; Razavi Khorasan Province, 9177948974, Mashhad, Iran.

Cite this article as: Palik M, Abdi K. The relationship between watching sport events and spectators' engagement in physical activities. Pedagogics, psychology, medical-biological problems of physical training and sports, 2018;22(6):320-327. https://doi. org/10.15561/18189172.2018.0607

The electronic version of this article is the complete one and can be found online at: http://www.sportpedagogy.org.ua/index.php/PPS/issue/archive

This is an Open Access article distributed under the terms of the Creative Commons Attribution License, which permits unrestricted use, distribution, and reproduction in any medium, provided the original work is properly cited (http://creativecommons.org/licenses/by/4.0/deed.en).

Received: 06.05.2018

Accepted: 15.06.2018; Published: 22.12.2018 